\title{
On the stress state of a pressurised pipe with an initial thickness variation, subjected to non-homogeneous internal corrosion
}

\author{
Shixiang Zhao ${ }^{1}$ and Yulia Pronina, ${ }^{1, *}$ \\ ${ }^{1}$ Saint Petersburg State University, Department of Computational Methods in Continuum Mechanics, \\ 199034 Universitetskaya nab. 7/9, St. Petersburg, Russia
}

\begin{abstract}
The paper concerns 2D problem of an elastic thick-walled pipe with an initial thickness variation, subjected to internal pressure and mechanochemical corrosion. The inner perimeter of the pipe cross-section is elliptical, while the outer is circular. The linear Dolinskii corrosion kinetics model is used. In the general case, structural instability of initial boundary value problems with unknown evolving boundaries can cause the divergence of numerical procedures when modelling the processes under study. It is observed that the attempts to circumvent the divergence of numerical procedure can suppress the manifestation of mechanochemical effect and yield inaccurate results. Thus, it is necessary to find a compromise between competing computational processes. Calculations revealed that the variation of the initial pipe wall thickness within the acceptable pipe wall tolerance can noticeably accelerate the growth of stresses and, consequently, reduce the durability of the pipe. The applicability of analytical solutions for a perfect circular pipe with a reduced thickness, equal to the minimum thickness of the imperfect pipe, to the case under study is also discussed.
\end{abstract}

\section{Introduction}

Many pipelines are subjected to pressure and chemical exposure. Simultaneous action of mechanical loads and aggressive environments can cause mechanochemical corrosion. The term "mechanochemical corrosion" was introduced by E.M. Gutman with regard to general corrosion facilitated by stress, to be more precise, for anodic dissolution of elastically deformed metals [1]. The rate of corrosion can often grow almost linearly with stress $[1,2,3]$. The linear corrosion kinetics equation can also be considered as an approximation of any other dependence. In cases of complex stress state, different authors used the von Mises stress, maximum normal stress, or mean stress as the equivalent stress in corrosion kinetics equations $[3,4,5,6,7]$. According to [8], the best correlation with experimental results is observed when the maximum in absolute value principal stress is used. However, when the effect of the strain sign takes place $[8,9]$ and the principal stresses have different signs, additional experimental investigations are required. These situations can occur in pipes subjected to both internal and external pressure.

A number of closed-form solutions for determining the stress state of structural elements subjected to uniform mechanochemical corrosion have been obtained by the authors of $[2,3,4,5,6,9,10,11,12,13]$ and others. More complex problems for pipeline elements when the equivalent stresses on their inner and outer surfaces are different have been studied in $[14,15,16$, 17]. Problems taking into account nonlinear effects and/or concerning non-uniform wear are usually solved by combined numerical and analytical methods $[4,7,10$, $18,19,20,21,22,23]$. In particular, paper [19] addresses the problem of a thin-walled cylindrical pipe with non-circular cross-section and variable wall thickness under external pressure; while paper [22] deals with a pipe with variable wall thickness under its own weight. All the mentioned solutions are based on certain hypotheses which significantly simplify the calculation process. When any simplifications cannot be accepted, structural instability of problems with evolving boundaries may cause the divergence of numerical procedures. Computational complexity in such case increases dramatically [24].

The present paper is devoted to determining the stress state of a pressurised thick-walled pipe with an initial wall thickness variation under conditions of internal mechanochemical corrosion. The inner perimeter of the pipe cross-section at the initial time is elliptical, while the outer is circular. The problem is solved without an assumption that the shape of the inner perimeter during the corrosion process remains elliptical (as it was assumed in $[25,26])$. The hypotheses of thin shells theory $[19,27]$ are not applicable in this case as well. Stress state of the corroding pipe at every time-step is determined by finite element method (FEM), after that the new shape of the inner surface is specified. As noted in [28], lack of stability can lead to bad results obtained with the FEM, and the combination of numerical and semi-analytical methods is more effective. Nevertheless, for the problem under study, we could circumvent the

\footnotetext{
* Corresponding author: y.pronina@spbu.ru
} 
divergence of numerical procedure and obtain results that are in good agreement with analytical solution for the mechanochemical corrosion of a circular concentric pipe under pressure [16]. The applicability of this analytical solution for a perfect pipe of the reduced thickness, equal to the minimum thickness of the imperfect pipe, to the problem under study is also discussed.

\section{Formulation of the problem}

Consider a linearly elastic isotropic long thick-walled tube subjected to constant internal pressure $p$. The outer surface of the tube is a circular cylinder with a radius $R$. The inner surface at the initial time $t=0$ is an elliptical cylinder with the cross-section semi-axes $A$ and $B$; $(A>B)$. The central axes of both cylinders coincide with each other. The difference $A-B$ is quite small and satisfies the following requirements imposed on the outof-roundness and thickness variation for the pipe:

$$
\begin{gathered}
{[2(A-B) /(\mathrm{A}+\mathrm{B})] 100 \%<1.5 \%,} \\
{[(\mathrm{~A}-B) /(R-(A+B) / 2)] 100 \%<10 \% .}
\end{gathered}
$$

The tube is subjected to internal mechanochemical corrosion defined as general dissolution of the material with a rate $v$ proportional to the maximum normal stress, $\sigma_{1}$, at a corresponding point $s$ of the inner surface $[2,8]$ :

$$
v(s)=\mathrm{d} h(s) / \mathrm{d} t=a+m \sigma_{1}(s) .
$$

Here, $a$ and $m$ are empirically determined constants of the corrosion kinetics; $\mathrm{d} h(s)$ is an increment of the depth of material dissolution (during a time interval $\mathrm{d} t$ ) at a position $s$ on the inner surface in the direction of its normal. The maximum principal stress, $\sigma_{1}$, acts along the cylinder guide on the planes perpendicular to this guide. Since the principal stress is not uniform along the crosssection, the dissolution process is not equal-rate as well. Then, the shape of the inner surface changes, causing, in turn, continuous changes in the principal stresses.

The action of the ends of the cylinder is not taken into account.

The purpose of the study is to assess the effect of the variation of the pipe wall thickness on the stress growth during the mechanochemical corrosion process and on the lifetime of the pipe.

\section{Method of solution}

The problem is reduced to the plane strain problem for the cross-section of the tube loaded with a constant normal pressure $p$ on the evolving internal contour denoted by $L$. The evolution of $L$ is affected by the elastic stress along this contour, which, in turn, depends on the shape of the evolving surface.

Since this initial boundary value problem with unknown boundary $L$ cannot be solved by existing software packages, a new program was created in Matlab environment. The algorithm consists of the following basic steps. (I) At initial time $t=0$, the shape of inner contour $L$ is an ellipse with semi-axes $A$ and $B$, the outer contour is a circle with a radius $R$. Normal pressure $p$ is applied on the inner contour. The outer surface is free. (II) The principal stress $\sigma_{1}$ along the contour $L$ is determined by solving the conventional boundary value problem stated in (I) by FEM. (III) For a given time increment $\mathrm{d} t$, the new shape of contour $L$ is determined by Eq. (3). Normal pressure $p$ is applied on the evolved contour $L$. The outer contour remains constant and free. (IV) The stress $\sigma_{1}$ along the updated contour $L$ is determined by solving the new boundary value problem stated in (III) by FEM. Then the steps (III) and (IV) repeat for the next time steps.

The choice of the time step is not complicated and may be performed by conventional procedures, although it must be consistent with the step in space. The choice of the optimal number of finite elements along the evolving contour requires a lot of additional investigation. On the one hand, a decrease in the grid step leads to a decrease in the approximation error of the smooth boundary and more accurate values of stress for a static problem. On the other hand, an increase in the number of finite elements causes accumulation of the rounding error and a poor conditionality of the resolving system of equations, as well as a significant increase in computational cost. Since we have to repeat the computational cycle several hundred times, an unrecoverable error of the method (in stress values) accumulates and leads to inadequate results. The attempts to smooth out computation errors can suppress the manifestation of the mechanochemical effect and yield inaccurate results as well. Thus, a number of computational experiments and special technics were implemented to arrive at a compromise between various competing computational processes. The developed algorithm was verified by comparing the calculation results for a concentric circular tube with the results obtained by the use of analytical solution for such problem [16] and by various passages to the limiting cases.

\section{Calculation results}

Several examples are considered for the pipes with an initial thickness variation within the acceptable ovality and pipe wall tolerance satisfying conditions (1) and (2). Graphs of the changes (with time) in the maximum principal stress $\sigma_{1}$ on the inner surface of the pipe are demonstrated on Figs. $1-3$. For all the figures, $R=120$ $\left[l_{c}\right], B=100\left[l_{c}\right]$, and the corrosion kinetics constants $a=0.1\left[l_{c} / t_{c}\right]$ and $m=a / 200\left[l_{c} /\left(t_{c} p_{c}\right)\right]$. Here, $l_{c}, t_{c}$, and $p_{c}$ are certain units of length, time and stress.

Solid lines are used for the stresses in imperfect tubes (when $A>B$ ), while dashed lines for perfect (i.e. circular concentric with $r=A=B$ ) tubes. All the graphs for the perfect tubes were built by the use of both analytical solution [16] and the developed program. The results are completely matched.

Figure 1 shows the stress growth at the vertices (e.g., node 121 from Fig. 2) of the contour $L$ with the initial coordinates $x_{0}= \pm A, y_{0}=0$ (solid lines 4 ) and at the 
points (e.g., node 1) with the initial coordinates $x_{0}=0, y_{0}$ $= \pm B$ (solid lines 1) for imperfect tube with $A=100.75$ $\left[l_{c}\right]$, and stress at the inner surface (at any point) of the perfect tubes with the inner radii $r=B$ (dashed lines 2) and $r=A$ (dashed lines 3). Here, axes $0 x$ and $0 y$ are directed along the main axes of the ellipse. Grey curves correspond to $p=5\left[p_{c}\right]$ and black curves to $p=10\left[p_{c}\right]$.

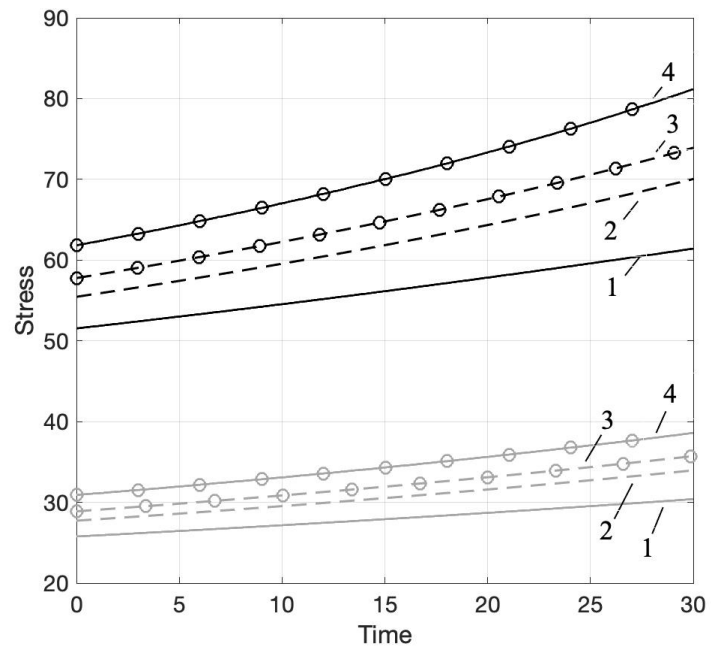

Fig. 1. Stress growth with time at $p=5$ (grey lines) and $p=10$ (black lines). $A=100.75$ and $B=100$ for imperfect pipe (solid lines) and $r=100.75$ (dashed lines 3); $r=100$ (dashed lines 2) for perfect pipes. $R=120$.

As one can see, the greater the initial stress, the higher the rate of its growth. The most noticeable manifestation of the effect under study is observed at high pressures, as demonstrated in Fig. 2 for $p=40\left[p_{c}\right]$.

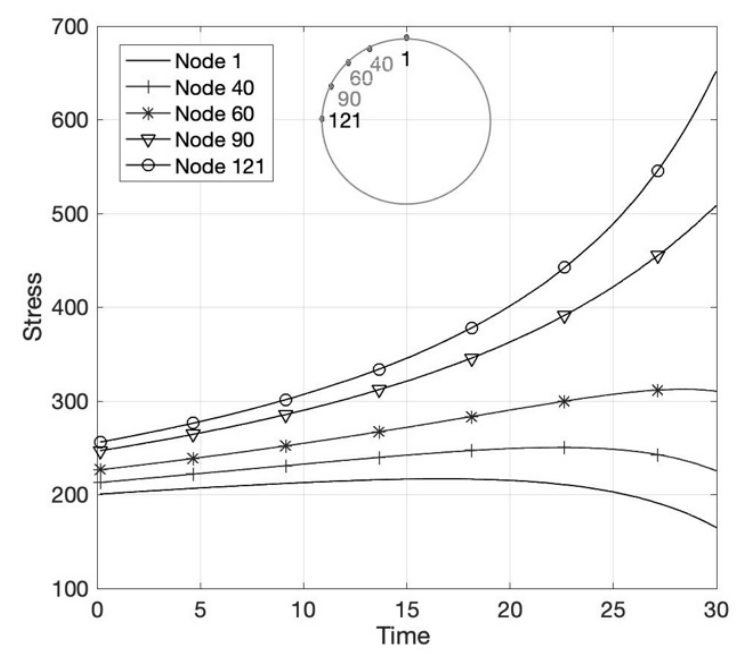

Fig. 2. Stress growth with time for $p=40$ at different nodes on the inner surface of imperfect pipe with $A=101, B=100$, and $R=120$.

The greatest increase in the stress occurs at the vertices of the inner surface (at node 121), which leads to the stress concentration in its vicinity. This may initiate localisation of corrosion.

Figure 3 shows the growth of the stress $\sigma_{1}$ with time for $p=40\left[p_{c}\right]$ at the vertices (nodes 121) of imperfect pipes (black solid lines) for different values of the initial inner perimeter ovality at $A=100.5 ; 101 ; 101.5$; and 102 $\left[l_{c}\right]$. For comparison, the curves for the perfect pipes with $r=A$ and $r=B=100\left[l_{c}\right]$ are shown (grey dashed lines).

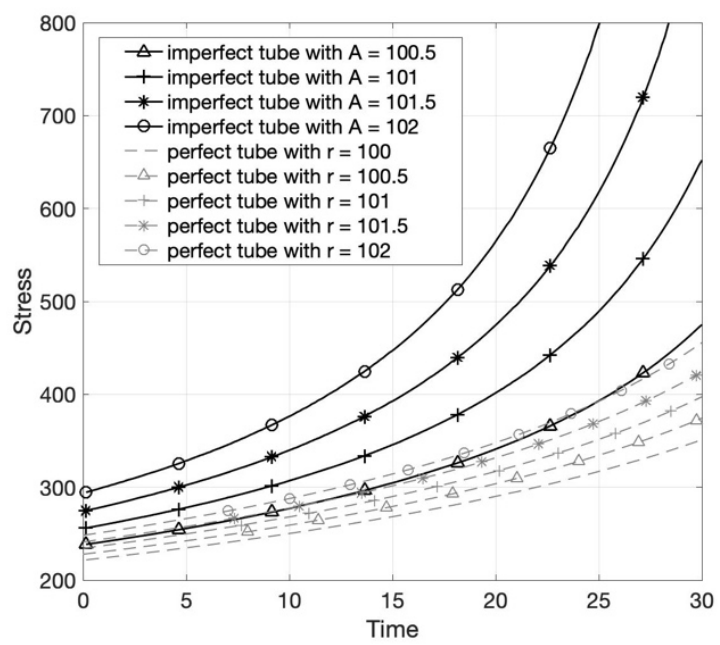

Fig. 3. Stress growth with time for $p=40$ at the vertices (nodes 121) of imperfect (black solid lines) and perfect (grey dashed lines) pipes of various sizes. $B=100$ and $R=120$.

As one can see, the maximum stress growth for pipes with an initial wall thickness variation is much higher than for perfect pipes with a reduced thickness equal to the minimum thickness of the imperfect pipe. This means that the acceleration of the maximum stress growth can be induced not only by local smooth thinning of the pipe wall, but also by its local thickening.

If we define lifetime of the pipe as the time at which an effective stress reaches a certain limit (e.g., corresponding to loss of stability [29], plastic yielding $[16,30]$ or brittle fracture [7]), then we can see that the strong mechanochemical effect can dramatically reduce the life of the pipe due to even slight ovality of its inner surface.

Add that the presence of minor surface and subsurface defects $[31,32,33,34,35,36]$ can result in a high stress gradient, which can cause electrochemical heterogeneity and localisation of the dissolution process. The results presented in the paper do not concern the localisation of corrosive wear.

\section{Conclusion}

The problem of determining the stress state of a pressurised thick-walled pipe with an initial wall thickness variation under conditions of internal mechanochemical corrosion was considered. The results of the calculations turned out to be very sensitive to the finite element method errors. At the same time, the attempts to circumvent the divergence of numerical procedure often suppressed the manifestation of mechanochemical effect. Thus, it was necessary to find a compromise between competing computational processes. 
Calculations revealed that even a slight variation (both thinning and thickening) of the initial pipe wall thickness within the acceptable pipe wall tolerance can noticeably accelerate the growth of maximum stresses and, consequently, reduce the durability of the pipe, especially when the initial stresses are high enough. The stress concentration occurs in the vicinity of pipe wall thinning, while stresses along the surface with a minimum curvature can even go down.

\section{References}

1. E.M. Gutman, Mechanochemistry of Metals and Corrosion Protection (Metallurgiya, Moscow, 1974) [in Russian]

2. V.M. Dolinskii, Chem. Petroleum Eng. 3(2), 96-97 (1967).

3. I.G. Ovchinnikov, Y.M. Pochtman, Mater. Sci. 27 (2), 105-116 (1992).

4. V.G. Karpunin, S.I. Kleshchev, M.S. Kornishin, Proceedings, 10th All-Union Conference of the Theory of Shells and Plates 1, 166-174 (Tbilisi, 1975) [in Russian]

5. M.S. Kornishin, V.G. Karpunin, Proceedings of a Seminar on Shell Theory 6(2), 58-66 (Kazan, 1975) [in Russian]

6. E.M. Gutman, R.S. Zainullin, A.T. Shatalov, R.A. Zaripov, Strength of gas industry pipes under corrosive wear conditions (Nedra, Moscow, 1984) [in Russian]

7. V.V. Petrov, I. G. Ovchinnikov, Y. M. Shihov, Calculation of structural elements, interacting with aggressive media (Saratov State University, Saratov, 1987) [in Russian]

8. P.A. Pavlov, B.A. Kadyrbekov, V.A. Kolesnikov, Strength of Steels in Corrosive Environments, (Nauka, Alma-Ata, 1987) [in Russian]

9. A.I. Rusanov, Russ. Chem. Rev. 85(1), 1-13 (2016)

10. G.A. Naumova, I.G. Ovchinnikov, Strength calculations of compound frameworks and pipeline structures with taking into account corrosion damages (Saratov State University, Saratov, 2000) [in Russian]

11. I. Elishakoff, G. Ghyselinck, Y. Miglis, J. Appl. Mech. 79(2), 021013 (2012)

12. M.M. Fridman, I. Elishakoff, Ocean Systems Eng. 5(1), 21-30 (2015)

13. E. Gutman, R. Bergman, S. Levitsky, Corr. Sci. 111, 212-215 (2016)

14. Y.G. Pronina, Russ. Metall. (Metally) 2010(4), 361364 (2010)

15. Y.G. Pronina, Proceedings of the 4th International Conference on Computational Methods for Coupled Problems in Science and Engineering, COUPLED PROBLEMS 2011, 1408-1415 (2011)
16. Y. Pronina, Int. J. Solids Struct. 50(22-23), 3626-33 (2013)

17. Y. Pronina, O. Sedova, M. Grekov, T. Sergeeva, Int. J. Eng. Sci. 130, 115-28 (2018)

18. V.M. Dolinskii, Dynamics and strength of machines 22, 81-84 (1975)

19. E.M. Gutman, J. Haddad, R. Bergman, Thin-Walled Struct. 43, 23-32 (2005)

20. V.V. Kabanin, G.A. Naumova, I.I. Ovchinnikov, Strength of shells of revolution subjected to corrosive wear in a nonuniform temperature field (IACE of VSTU, Volgograd, 2007) [in Russian]

21. M.M. Fridman, J. Theor. Appl. Mech. 52(1), 129137 (2014)

22. I.A. Stareva, Y.G. Pronina, AIP Conference Proceedings 1959, 070034 (2018) DOI: $10.1063 / 1.5034709$

23. M. Poluektov, A.B. Freidin, Ł. Figiel, Int. J. Eng. Sci. 128, 44-62 (2018)

24. J.H. Prevost, T.J. Baker, J. Liang, Z. Suo, Int. J. Solids Struct. 38(30-31), 5185-5203 (2001)

25. Y. Pronina, Eur. J. Mech. A Solids 61, 357-63 (2017)

26. Y.G. Pronina, S.M. Khryashchev, Mater. Phys. Mech. 31(1-2), 52-5 (2017)

27. S.A Kabrits, E.P. Kolpak, 2015 International Conference on "Stability and Control Processes" in Memory of V.I. Zubov, SCP 2015 - Proceedings 7342171, 389-391 (2015)

28. D. Weisz-Patrault, A. Ehrlacher, N. Legrand, Int. J. Solids Struct. 94-95, 1-20 (2016).

29. I.V. Atavin, B.E. Melnikov, A.S. Semenov, N.V. Chernysheva, E.L. Yakovleva, Magaz. Civil Eng. 80(4), 48-61 (2018)

30. I.N. Izotov, N.P. Kuznetsov, B.E. Melnikov, A.G. Mityukov, S.V. Ponomareva, Proceedings of SPIE The International Society for Optical Engineering 5400, 244-257 (2004)

31. A.O. Bochkarev, M.A. Grekov, 2015 International Conference on "Stability and Control Processes" in Memory of V.I. Zubov, SCP 2015 - Proceedings 7342150, 367-370 (2015)

32. M.A. Grekov, S.A. Kostyrko, Int. J. Eng. Sci. 89, 61-74 (2015)

33. A.S. Semenov, S.G. Semenov, B.E. Melnikov, V.S. Tikhonov, Magaz. Civil Eng. 74(6), 78-101 (2017)

34. M.A. Grekov, T.S. Sergeeva, Y.G. Pronina, O.S. Sedova, Eng. Fract. Mech.186, 423-35 (2017)

35. R.L. Lapin,V.A. Kuzkin, M. Kachanov, Int. J. Eng. Sci. 124, 16-23 (2018)

36. S. Kostyrko, G. Shuvalov, Continuum Mech. Thermodynamics (2019) (published online). 